\title{
Metric Distance Ranking Technique for Fuzzy Critical Path Analysis
}

\section{*11 OLADEINDE; MH OLADEINDE $^{2}$ CA}

\author{
Production Engineering Department, Faculty of Engineering, University of Benin, Benin City, Nigeria \\ Email ${ }^{\prime}$ : moladeinde@uniben.edu (corresponding author) \\ Email': kallyama@yahoo.com
}

KEYWORDS: fuzzy, metric distance, critical path method, total float

\begin{abstract}
In this paper, fuzzy critical path analysis of a project network is carried out. Metric distance ranking technique is used to order fuzzy numbers during the forward and backward pass computations to obtain the earliest start, earliest finish, latest start and latest finish times of the project's activities. A numerical example is presented with a detailed step by step solution. The results of the numerical example presented are in agreement with published results in literature using other fuzzy ranking technique.@ JASEM
\end{abstract}

http://dx.doi.org/10.4314/jasem.v18i4.4

INTRODUCTION: In critical path method, the challenge facing the project manager is to determine the expected project completion time as well as activities which must be executed as planned if the promised completion date is to be realized. This is a very challenging job (Oladeinde and Oladeinde, 2014) as late completion in most cases attracts a penalty. It is not uncommon in the construction of industrial plants to provide performance incentives to contractors for early completion, quality work and adherence to safety rules and regulations (Bubshait, 2003). The project manager's dilemma to avoid disincentives arising from late completion of projects escalates when he has to deal with projects whose activities durations are uncertain and can only be expressed in vague form using linguistic expressions. In many practical situations, specifically when an activity is being executed for the first time, the project manager is unable to specify a deterministic value for the time to execute the activity. The successful implementation of critical path method under such circumstance becomes difficult if not entirely impossible. In order to address such vagueness in activity durations, Program Evaluation and Review Technique based on probability theory has been applied The originators of PERT modeled each activity duration as a stochastic variable based on the beta distribution and proposed an approximate method to calculate the expectation and the variance of the makespan of the project (Yakhchali, 2011). The originators of PERT estimated the activity duration using three time estimates namely pessimistic, optimistic and most likely times. The validity of this approximation method has been the subject of intense debate among researchers. In order to address the shortcomings of PERT, fuzzy theory has been advanced as a useful tool for dealing quantitatively with imprecise activity durations. A number of authors have participated in the use of fuzzy theory for critical path analysis. Khalaf (2013) applied fuzzy theory to find fuzzy total float, fuzzy free float and fuzzy independent float for a project network with activity duration modeled as triangular fuzzy numbers. Oladeinde and Itsisor (2013) obtained the fuzzy early start, fuzzy early finish, fuzzy latest start, fuzzy latest finish as well as the critical activities in a project network with triangular activity duration. In crisp environment, the comparison of numbers is carried out using normal arithmetic operations. In fuzzy environment, fuzzy number comparison is carried out using ranking technique. Different ranking techniques available which have been used by different authors include but is not limited to Centroid method, center of gravity method, Decision maker risk attitude and Metric distance technique.

In this paper, the metric distance ranking technique is used to solve a fuzzy critical path problem with triangular representation of activity duration. The method is illustrated with a numerical example with detailed step by step solution. 
Metric Distance Ranking Technique for Fuzzy

FUZZY FUNDAMENTALS: Let $R$ be the space of real numbers. A fuzzy set of numbers is a set of ordered pairs $\left\{\left(x, \mu_{A}(x)\right) \mid x \in R\right\}$, where $\mu_{A}(x): R \rightarrow[0,1]$ and is upper semi continuous. $\mu_{A}(x)$ is called the membership function of the fuzzy set. A convex fuzzy set is a fuzzy set in which Eq. (1) and (2) holds

$\forall x, y \in R, \forall \lambda \in[0,1]$

$\mu_{A}(\lambda x+(1-\lambda) y) \geq \min \left[\mu_{A}(x), \mu_{A}(y)\right]$

A fuzzy set $A$ is called positive if its membership function is such that $\mu_{A}(x)=0, \forall x \leq 0$.

A triangular fuzzy set $A$ is a convex fuzzy set which is defined as $A=\left(x, \mu_{A}(x)\right)$ where

$\mu_{A}(x)= \begin{cases}\frac{x-a}{b-a} & \mathrm{a} \leq \mathrm{x} \leq \mathrm{b} \\ \frac{c-a}{c-b} & \mathrm{~b} \leq \mathrm{x} \leq \mathrm{c} \text { (3) } \\ 0 & \text { otherwise }\end{cases}$

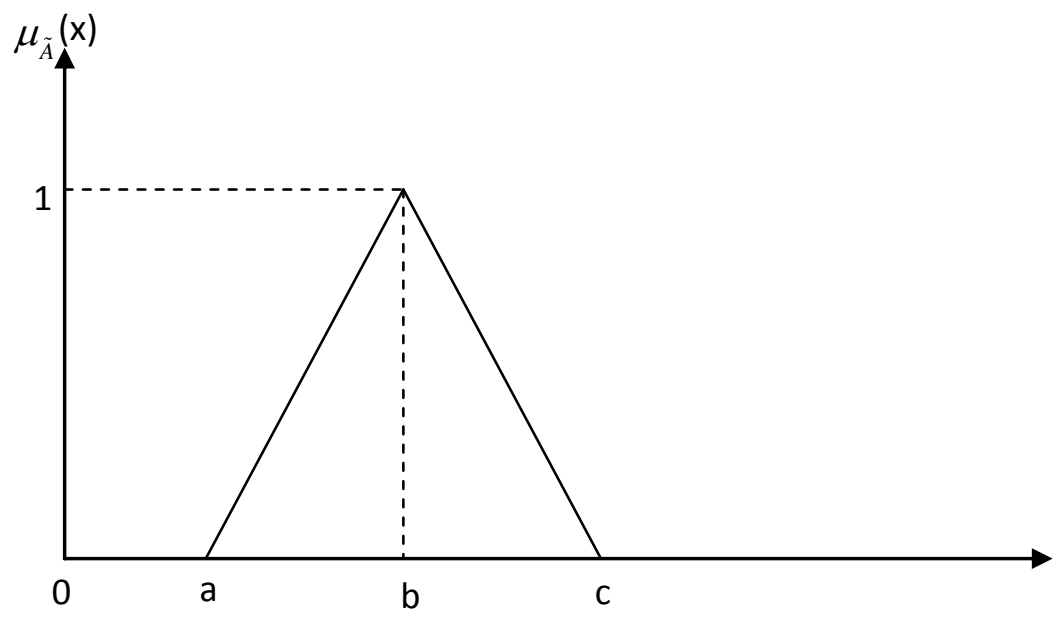

Fig 1: A triangular fuzzy number

The triangular fuzzy set $A$ is given by the set of numbers $(a, b, c)$ where $0 \leq a \leq b \leq c$

Fuzzy Set Arithmetic Operations :Given two triangular fuzzy sets $A=\left[a_{1}, b_{1}, c_{1}\right]$ and $B=\left[a_{2}, b_{2}, c_{2}\right]$, fuzzy arithmetic and subtraction are implemented as follows

$\tilde{A} \oplus \tilde{B}=\left[a_{1}, b_{1}, c_{1}\right] \oplus\left[a_{2}, b_{2}, c_{2}\right]=\left[\left(a_{1}+a_{2}\right),\left(b_{1}+b_{2}\right),\left(c_{1}+c_{2}\right)\right]$
$\tilde{A} \Theta \tilde{B}=\left[a_{1}, b_{1}, c_{1}\right] \Theta\left[a_{2}, b_{2}, c_{2}\right]=\left[\left(a_{1}-c_{2}\right),\left(b_{1}-b_{2}\right),\left(c_{1}-a_{2}\right)\right]$

Fuzzy Cpm Forward Pass :Consider a project network with activity times $t_{i j}$ where the vertices $(V)$ represent the set of node numbers and the direct edges represent the activities. An activity is represented by one and only one arrow with its tail event at node $i$ and its head event at node $\mathrm{j}(i, j \in V)$ where $i<j$.In fuzzy forward pass, we compute the earliest fuzzy time $\left(E_{j}\right)$ of event $j$, the earliest fuzzy start time (ES) of activity $(i, j)$, the earliest fuzzy finish time $(E F)$ of activity $(i, j)$ and the fuzzy completion $\left(T_{F}\right)$ time of the 
project. The earliest fuzzy time of event $j$ can be obtained by implementing the CPM forward pass methodology in the fuzzy environment using the expression $\tilde{E}_{j}=\max _{i \in p(j)}\left[\tilde{E}_{i} \oplus \tilde{t}_{i j}\right] \quad \mathrm{i} \in p(j) \neq \phi(6)$ In the expression above, $i \in p(j)$ denotes the set of activities $i$ which precedes the node $j$. When there are no proceeding events $(p(j)=\phi)$ to the event under inspection which corresponds uniquely to the starting node in the project, the fuzzy time of starting the project $\left(T_{S}\right)$ is given as $(0,0,0)$. The fuzzy earliest finish time of an activity is given by

$$
E F_{i j}=E S_{i j}+t_{i j}
$$

Fuzzy Cpm Backward Pass: In crisp environment, backward pass is used to calculate the latest event times as well as the latest finish times and fuzzy latest start time of activities. In fuzzy environment, we calculate the fuzzy latest time of event $i\left(L_{i}\right)$, the fuzzy latest finish time $\left(L F_{i j}\right)$ of activity $(i, j)$ and the fuzzy latest start time $\left(L S_{i j}\right)$ of activity $(i, j)$. The fuzzy completion time of a project is the maximum of the earliest finish times of all the activities which have no successors in the project. The maximum earliest finish times is obtained by means of centroid ranking of fuzzy numbers. The fuzzy latest start time of an activity is given by

$$
L S_{i j}=L F_{i j} \Theta t_{i j}(8)
$$

The fuzzy latest time of a node is obtained by finding the minimum of the latest start times of all activities which originate from the node in question

Total Float/Slack: The fuzzy total float of an activity is found after obtaining the fuzzy activity times namely, latest finish time and earliest start times. The fuzzy total float is computed using the equation

$$
T F=L F \Theta E S \Theta t \quad(9)
$$

Equation 6 is implemented using fuzzy arithmetic operations defined previously.

Fuzzy Number Ranking: Ranking is used to order fuzzy numbers as normal comparison carried out in crisp environment is impossible. A number of fuzzy ranking methods have been used in the literature. The Decision Makers risk attitude index ranking technique has been used by a number of authors namely Oladeinde and Oladeinde (2014) and Liang and Han (2004). Maximizing-Minimizing points have been used by Salashshour et al (2011) for ranking fuzzy numbers. Fuzzy number ordering has been achieved by Karimirad et al (2013).based on upper and lower bounds.

\section{METRIC DISTANCE RANKING}

The metric distance ranking technique was proposed by Chen and Cheng (2005) to order fuzzy numbers. Let two fuzzy numbers A and B be defined as follows:

$$
\begin{aligned}
& f_{A}(x)=\left\{\begin{array}{l}
f_{A}^{L}(x), x<m_{A} \\
f_{A}^{R}(x), x<m_{A}
\end{array}\right. \\
& f_{B}(x)=\left\{\begin{array}{l}
f_{B}^{L}(x), x<m_{B} \\
f_{B}^{R}(x), x<m_{B}
\end{array}\right.
\end{aligned}
$$

In Equations (1) and (2), $m_{A}$ and $m_{B}$ represent the mean of fuzzy numbers $A$ and B respectively. The metric distance between A and B can be calculated as follows (Sireesha and Shanker, 2010)

$$
D(A, B)=\left[\int_{0}^{1}\left(g_{A}^{L}(y)-g_{B}^{L}(y)\right)^{2} d y+\int_{0}^{1}\left(g_{A}^{R}(y)-g_{B}^{R}(y)\right)^{2} d y\right]
$$

In Equation (3) $g_{A}^{L}, g_{A}^{R}, g_{B}^{L}$ and $g_{A}^{R}$ are the inverse functions of $f_{A}^{L}, f_{A}^{R}, f_{B}^{L}$ and $f_{B}^{R}$ respectively. Setting the fuzzy number $\mathrm{B}=0$, the metric distance between $\mathrm{A}$ and $\mathrm{B}$ can be calculated as follows 


$$
D(A, 0)=\left[\int_{0}^{1}\left(g_{A}^{L}(y)\right)^{2} d y+\int_{0}^{1}\left(g_{A}^{R}(y)\right)^{2} d y\right]
$$

The larger the value of $\mathrm{D}(\mathrm{A}, 0)$, the better the ranking of $\mathrm{A}$ (Shanker et al, 2010). A triangular fuzzy number can be represented as a symmetry fuzzy number having two parameters namely, standard deviation $(\sigma)$ and mean $(\mu)$. The membership function of the symmetry fuzzy number A in terms of its standard deviation and mean is given as

$$
f_{A}(x)=\left\{\begin{array}{l}
\frac{x-(\mu-\sigma)}{\sigma}, \text { if }(\mu-\sigma) \leq x \leq \mu \\
\frac{(\mu+\sigma)-x}{\sigma}, \text { if } \mu \leq x \leq \mu+\sigma
\end{array}\right.
$$

For a triangular fuzzy number $\left(a_{1}, a_{2}, a_{3}\right)$, the mean and standard deviation can be determined using the following:

$$
\begin{aligned}
& \sigma=\frac{a_{3}-a_{1}}{2} \\
& \mu=\frac{a_{1}+2 a_{2}+a_{3}}{4}
\end{aligned}
$$

The inverse of $f_{A}^{L}$ and $f_{A}^{R}$ are given as

$$
\begin{aligned}
& g_{A}^{L}(y)=(\mu-\sigma)+\sigma y \\
& g_{A}^{R}(y)=(\mu+\sigma)-\sigma y
\end{aligned}
$$

Deffuzification Of Fuzzy Set: Deffuzification of fuzzy set is used to obtain crisp values which represent uncertain data. When fuzzy number is converted into a crisp number, it becomes easy to make comparison between two or more fuzzy numbers and consequently facilitate decision making. A number of deffuzification methods are in use for different types of fuzzy numbers. For a triangular fuzzy number $A=(a, b, c)$, we can obtain the crisp value representing the fuzzy number by finding the median using the expression

$$
M_{A}=\frac{a+2 b+c}{4}
$$

For the purpose of finding the critical activities in a project, Eq. (10) can be used to defuzzify the fuzzy total float of the activity. A critical activity is one whose total float is equal to zero.

Numerical Example : The data presented in Table 1 is for a fizzy project network solved by Elizabeth and Sujatha (2013) using the Decision Makers' risk index ranking. We propose a solution to the problem using metric distance ranking Technique.

Table 1: Project Activities and their fuzzy Durations

\begin{tabular}{ll}
\hline Activity & Fuzzy Activity Time \\
\hline $\mathbf{1 - 2}$ & $(2,3,4)$ \\
$\mathbf{1 - 3}$ & $(1,3,4)$ \\
$\mathbf{2 - 4}$ & $(1,3,5)$ \\
$\mathbf{3 - 5}$ & $(1,2,3)$ \\
$\mathbf{3 - 6}$ & $(2,5,7)$ \\
$\mathbf{4 - 6}$ & $(3,4,6)$ \\
$\mathbf{4 - 7}$ & $(3,4,5)$ \\
$\mathbf{5 - 7}$ & $(1,4,5)$ \\
$\mathbf{6 - 8}$ & $(2,5,6)$ \\
$\mathbf{7 - 8}$ & $(3,4,7)$ \\
\hline
\end{tabular}

The precedence diagram of the project network is presented in Figure 2

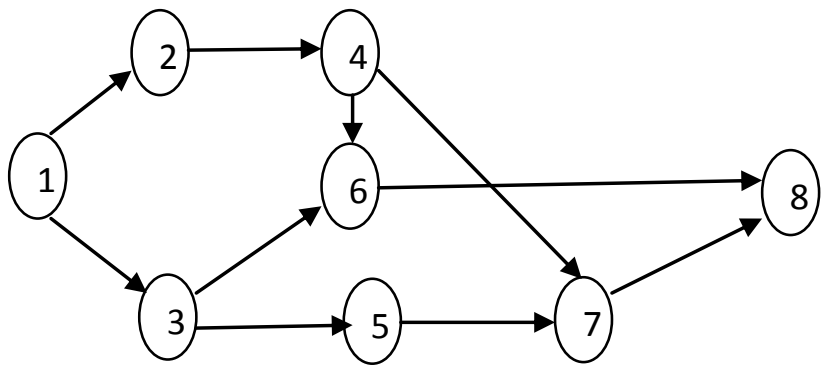

Fig 2: Network diagram of numerical example 
he earliest start times as well as the earliest finish times of the activities starting the project are obtained using forward pass method described previously.

$$
\begin{array}{lrl}
E S_{12}=0,0,0 & E F_{12}=(0,0,0) \oplus(2,3,4)=(2,3,4) \\
E S_{13}=0,0,0 & E F_{13}=(0,0,0) \oplus(1,3,4)=(1,3,4) \\
E S_{24}=E F_{12}=(2,3,4) & E F_{24}=(2,3,4) \oplus(1,3,5)=(3,6,9) \\
E S_{36}=E F_{13}=(1,3,4) & E F_{36}=(1,3,4) \oplus(2,5,7)=(3,8,11) \\
E S_{35}=E F_{13}=(1,3,4) & E F_{35}=(1,3,4) \oplus(1,2,3)=(2,5,7) \\
E S_{46}=E F_{24}=(3,6,9) & E F_{46}=(3,6,9) \oplus(3,4,6)=(6,10,15)
\end{array}
$$

The earliest start time of activity 6-8 can be obtained by finding the metric distance ranking of the earliest finish times of activity 4-6 and activity 3-6. The metric distance ran king of the earliest finish time of activity 4-6 is calculated as shown below

$$
\begin{aligned}
& D\left(E F_{46}, 0\right)=\int_{0}^{1}\left(\left(\frac{41}{4}-\frac{9}{2}\right)+\frac{9 y}{2}\right) d y+\int_{0}^{1}\left(\left(\frac{41}{4}+\frac{9}{2}\right)-\frac{9 y}{2}\right) d y \\
& D\left(E F_{46}, 0\right)=223.63
\end{aligned}
$$

The metric distance ranking of the earliest finish time of activity 3-6 is calculated as:

$$
\begin{aligned}
& D\left(E F_{36}, 0\right)=\int_{0}^{1}\left(\left(\frac{30}{4}-4\right)+4 y\right) d y+\int_{0}^{1}\left(\left(\frac{30}{4}+4\right)-4 y\right) d y \\
& D\left(E F_{36}, 0\right)=123.17
\end{aligned}
$$

The earliest start time of activity 6-8 is the earliest finish time that gives the greater value of Equation 3. Since $D\left(E F_{46}, 0\right)>D\left(E F_{36}, 0\right), E S_{68}=(6,10,15)$

Having found the earliest start time of activity $6-8$, the earliest finish time is given as:

$$
\begin{array}{ll}
E F_{68}=(6,10,15) \oplus(2,5,6)=(8,15,21) \\
E S_{35}=E F_{13}=(1,3,4) & E F_{35}=(1,3,4) \oplus(1,2,3)=(2,5,7) \\
E S_{57}=E F_{35}=(2,5,7) & E F_{57}=(2,5,7) \oplus(1,4,5)=(3,9,12) \\
E S_{47}=E F_{24}=(3,6,9) & E F_{47}=(3,6,9) \oplus(3,4,5)=(6,10,14)
\end{array}
$$

The earliest start time of activity 7-8 can be found by finding the greater between $E F_{47}$ and $E F_{57}$. The metric distance ranking of $E F_{47}$ can be calculated as:

$$
\begin{aligned}
& D\left(E F_{47}, 0\right)=\int_{0}^{1}(6+4 y) d y+\int_{0}^{1}(14-4 y) d y \\
& D\left(E F_{47}, 0\right)=210.67 \\
& D\left(E F_{57}, 0\right)=\int_{0}^{1}\left(\left(\frac{33}{4}-\frac{9}{2}\right)+\frac{9}{2} y\right) d y+\int_{0}^{1}\left(\left(\frac{33}{4}+\frac{9}{2}\right)-\frac{9}{2} y\right) d y \\
& =D\left(E F_{57}, 0\right)=149.63
\end{aligned}
$$

The earliest start time of activity 7-8 is the earliest finish time that gives the greater value of Equation 3. Since $D\left(E F_{47}, 0\right)>D\left(E F_{57}, 0\right), E S_{78}=(6,10,14)$

The earliest finish time of activity 7-8 is therefore

$$
\mathrm{EF}_{78}=(9,14,21)
$$

The backward pass computations are now carried out to obtain the latest times of the activities in the project. First, we determine the completion time of the project which automatically becomes the latest finish times of the terminal activities in the project namely, activity 6-8 and activity 7-

8. The metric distance ranking values of $\mathrm{EF}_{78}$ is 444.5 while that for $\mathrm{EF}_{68}$ is 463.29 , the latest finish times of activities 6-8 and 7-8 are therefore $(8,15$, 21)

The latest start time of activity 7-8 is the fuzzy difference between $\mathrm{LF}_{78}$ and the duration of the activity. 
$L S_{78}=(8,15,21) \Theta(3,4,7)=(1,11,18)$

The latest finish times of activities 4-7 and 5-7 are equal to the $(1,11,18)$. The latest start times of activities 4-7 and 5-7 are computed as

$L S_{47}=(1,11,18) \Theta(3,4,5)=(-4,7,15), L S_{57}=(1,11,18) \Theta(1,4,5)=(-4,7,17)$

The latest start time of activity 6-8 is computed as

$L S_{68}=(8,15,21) \Theta(2,5,6)=(2,10,19)$

The latest start time of activity 4-6 is computed as the fuzzy difference between the latest finish time of activity 4-6 which equal $(2,10$, and 19$)$ and the duration of activity 4-6.

$L S_{46}=(2,10,19) \Theta(3,4,6)=(-4,6,16)$

The latest finish time of activity 2-4 is obtained after computing the metric distance ranking of the latest start times of activities 4-7 and 4-6.

$$
\begin{aligned}
& D\left(\mathrm{LS}_{46}, 0\right)=\int_{0}^{1}((6-10)+10 y) d y+\int_{0}^{1}((6+10)-10 y) d y=138.67 \\
& D\left(\operatorname{LS}_{47}, 0\right)=\int_{0}^{1}\left(\left(\frac{25}{4}-\frac{19}{2}\right)+\frac{19}{2} y\right) d y+\int_{0}^{1}\left(\left(\frac{25}{4}+\frac{19}{2}\right)-\frac{19}{2} y\right) d y=138.29
\end{aligned}
$$

Comparison of the metric distance ranking of the latest start times of activity 4-6 and activity 4-7 shows that the latest finish time of activity $2-4$ equals $(-4,6,16)$

The latest start time of activity 2-4 is computed as

$$
L S_{24}=(-4,6,16) \Theta(1,3,5)=(-9,3,15)
$$

The latest finish time of activity $3-6$ is the latest start time of activity $6-8$ which equals $(2,10$, and 19$)$. The latest start time of activity 3-6 can be calculated as follows:

$$
L S_{36}=(2,10,19) \Theta(2,5,7)=(-5,5,17)
$$

The latest finish time of activity 3-5 is the latest start time of activity 5-7 Therefore,

$$
L F_{35}=(-4,7,17)
$$

The latest start time of activity 3-5 is computed as

$$
L S_{35}=(-4,7,17) \Theta(1,2,3)=(-7,5,16)
$$

The latest finish time of activity 1-3 can be obtained by finding the metric distance ranking of the latest start time of activity 3-6 and activity 3-5. The latest start time which gives the smaller metric distance ranking is the latest finish time of activity 1-3. The metric distance ranking of $\mathrm{LS}_{37}$ is calculated as

$$
D\left(\mathrm{LS}_{35}, 0\right)=\int_{0}^{1}\left(\left(\frac{19}{4}-\frac{23}{2}\right)+\frac{23}{2} y\right) d y+\int_{0}^{1}\left(\left(\frac{19}{4}+\frac{23}{2}\right)-\frac{23}{2} y\right) d y=133.29
$$

The metric distance ranking of $\mathrm{LS}_{36}$ is computed as

$$
D\left(\mathrm{LS}_{36}, 0\right)=\int_{0}^{1}\left(\left(\frac{22}{4}-\frac{22}{2}\right)+\frac{22}{2} y\right) d y+\int_{0}^{1}\left(\left(\frac{22}{4}+\frac{22}{2}\right)-\frac{22}{2} y\right) d y=141.17
$$

Comparing the metric distance ranking of $\mathrm{LS}_{35}$ and $\mathrm{LS}_{36}$, the latest finish time of activity 1-3 is equal to $\mathrm{LS}_{35}$ since its metric distance ranking is smaller. The latest start time of activity 1-3 is therefore computed as

$$
L S_{13}=(-7,5,16) \Theta(1,3,4)=(-11,2,12)
$$

The latest finish of activity 1-2 is equal to the latest start time of activity 2-4. The latest start time of activity 1-2 is computed as

$$
L S_{12}=(-9,3,15) \Theta(2,3,4)=(-13,0,13)
$$

The summary of the result of the fuzzy critical path analysis is shown in Table 2 along with the fuzzy total float and defuzzified total float. The table shows that activities 1-2, 2-4, 4-6 and 6-8 have defuzzified total floats equal to zero. An activity which has a total float equal to zero is said to be critical. Therefore the critical path of the project network is 1-
2-4-6-8. The fuzzy completion time of the project is equal to $(8,15$, and 21$)$. The result obtained using the present method are in agreement with that obtained by Elizabeth and Sujatha (2013) who solved the same problem but used the Decision maker risk index method. 
Table 2: Fuzzy total float of each activity and the defuzzified total float values of each activity in the project network.

\begin{tabular}{llllllll}
\hline Activity & Duration & ES & EF & LS & LF & TF & Defuzzified TF \\
\hline $\mathbf{1 - 2}$ & $(2,3,4)$ & $(0,0,0)$ & $(2,3,4)$ & $(-13,0,13)$ & $(-9,3,15)$ & $(-13,0,13)$ & 0 \\
$\mathbf{1 - 3}$ & $(1,3,4)$ & $(0,0,0)$ & $(1,3,4)$ & $(-11,2,12)$ & $(-7,5,16)$ & $(-11,2,15)$ & 2 \\
$\mathbf{2 - 4}$ & $(1,3,5)$ & $(2,3,4)$ & $(3,6,9)$ & $(-9,3,15)$ & $(-4,6,16)$ & $(-13,0,13)$ & 0 \\
$\mathbf{3 - 5}$ & $(1,2,3)$ & $(1,3,4)$ & $(2,5,7)$ & $(-7,5,16)$ & $(-4,7,17)$ & $(-11,2,15)$ & 2 \\
$\mathbf{3 - 6}$ & $(2,5,7)$ & $(1,3,4)$ & $(3,8,11)$ & $(-5,5,17)$ & $(2,10,19)$ & $(-9,2,16)$ & 2.75 \\
$\mathbf{4 - 6}$ & $(3,4,6)$ & $(3,6,9)$ & $(6,10,15)$ & $(-4,6,16)$ & $(2,10,19)$ & $(-13,0,13)$ & 0 \\
$\mathbf{4 - 7}$ & $(3,4,5)$ & $(3,6,9)$ & $(6,10,14)$ & $(-4,7,15)$ & $(1,11,18)$ & $(-13,1,12)$ & 0.25 \\
$\mathbf{5 - 7}$ & $(1,4,5)$ & $(2,5,7)$ & $(3,9,12)$ & $(-4,7,17)$ & $(1,11,18)$ & $(-11,2,15)$ & 2 \\
$\mathbf{6 - 8}$ & $(2,5,6)$ & $(6,10,15)$ & $(8,15,21)$ & $(2,10,19)$ & $(8,15,21)$ & $(-13,0,13)$ & 0 \\
$\mathbf{7 - 8}$ & $(3,4,7)$ & $(6,10,14)$ & $(9,14,21)$ & $(1,11,18)$ & $(8,15,21)$ & $(-13,1,12)$ & 0.25 \\
\hline
\end{tabular}

Conclusion: Fuzzy critical path analysis of a project network has been carried out using metric distance ranking technique. The critical activities and hence the critical path of the project network has been obtained.

\section{REFERENCES}

Bubshait, A.A (2003) Incentive/disincentive contracts and its effects on industrial projects, International Journal of Project Management, vol 21 pp. 63-70

Chen LS, Cheng CH (2005) Selecting IS personnel using ranking fuzzy number by metric distance method. European Journal of Operational research 160 (3), pp 803- 820

Chanas and Zieli(nski (2001) Critical path analysis in the network with fuzzy activity times, Fuzzy Sets and Systems 122, pp 195204

Gin-Shuh Liang Tzeu-Chen Han (2004) Fuzzy Critical Path for a Project Network, Information and Management Sciences Volume 15, Number 4, pp. 29-40,

Karimirad, M, Jolai, F and Zowghi, M (2013) Ordering of fuzzy quantities based on upper and lower bounds, World of Sciences Journal special issue, pp $83-91$.

Khalaf, W.S (2013) Solving the Fuzzy Project Scheduling Problem Based on a Ranking Function, Australian Journal of Basic and Applied Sciences, 7(8): 806-811, 2013
Oladeinde M.H and Itsisor, D.O (2013) Application of Fuzzy theory to project scheduling with critical path method, J. Appl. Sci. Environ. Manage. Vol. 17 (1) 161-166

Oladeinde M.H and Oladeinde C.A (2014) An investigation into the Decision Makers Risk Attitude Index Technique for fuzzy critical Path Analysis, Nigeria Journal of Technology, vol 33, no 3, pp 345 - 350

Salahshour, S,Abbasbandy, $\quad$ S and Allahviranloo, T (2011) Ranking fuzzy Numbers using fuzzy maximizingminimizing points, EUSFLAT-LFA, pp 763-769

Shankar, N.R, Sireesha, V, Rao, K,S and Vani, N (2010) Fuzzy Critical Path Method Based on Metric Distance Ranking of Fuzzy Numbers, Int. Journal of Math. Analysis, Vol. 4, no. 20, pp. 995 - 1006

Sireesha, V and Shankar, N.R (2010) A New Approach to find Total Float time and

Critical Path in a fuzzy Project Network, International Journal of Engineering Science and Technology Vol. 2(4), pp. 600-609

Yakhchali, S.H (2011) A Simple Approach to Project Scheduling in Networks with Stochastic Durations, Proceedings of the World Congress on Engineering Vol I July 6 - 8, London, U.K 